\title{
VGRIS: Virtualized GPU Resource Isolation and Scheduling in Cloud Gaming
}

\author{
ZHENGWEI QI, JIANGUO YAO, CHAO ZHANG, MIAO YU, ZHIZHOU YANG \\ and HAIBING GUAN, Shanghai Jiao Tong University
}

To achieve efficient resource management on a graphics processing unit (GPU), there is a demand to develop a framework for scheduling virtualized resources in cloud gaming. In this article, we propose VGRIS, a resource management framework for virtualized GPU resource isolation and scheduling in cloud gaming. A set of application programming interfaces (APIs) is provided so that a variety of scheduling algorithms can be implemented within the framework without modifying the framework itself. Three scheduling algorithms are implemented by the APIs within VGRIS. Experimental results show that VGRIS can effectively schedule GPU resources among various workloads.

Categories and Subject Descriptors: C.4 [Performance of Systems]: Modeling Techniques, Measuring Techniques; C.0 [General]: System Architectures

General Terms: Design, Algorithms, Performance

Additional Key Words and Phrases: GPU, resource management, scheduling, cloud gaming

ACM Reference Format:

Zhengwei Qi, Jianguo Yao, Chao Zhang, Miao Yu, Zhizhou Yang, and Haibing Guan. 2014. VGRIS: Virtualized GPU resource isolation and scheduling in cloud gaming. ACM Trans. Architec. Code Optim. 11, 2, Article 17 (June 2014), 25 pages.

DOI: http://dx.doi.org/10.1145/2632216

\begin{abstract}
Extension of a Conference Paper: This article is an extension of a preliminary version appearing in Proceedings of the 22nd International ACM Symposium on High-Performance Parallel and Distributed Computing [Yu et al. 2013]. Compared with the prior work, we conduct three major revisions. In this article, we extend the framework with a set of API that can be leveraged to implement a variety of scheduling algorithms within the framework. Then, we rework all the evaluations. Instead of benchmarks such as 3DMark05 in the conference paper, the workloads are mainly selected from reality model games to prove the feasibility and practicality of the framework in real cloud gaming scenarios. Finally, we extend the evaluations by experimental results of VirtualBox. We compare the performance of VirtualBox and VMware, trying to explain why we select VMware, instead of VirtualBox, as the main virtualization platform. We also add the FPS results of the games running on heterogeneous platforms composed of VMware and VirtualBox to demonstrate that the framework is adapted to various VMs.
\end{abstract}

This work is supported by the Program for NSFC (Nos. 61272101, 61303013), the key program (No. 313035) of MOE, and the NRF Singapore under its CREATE program.

Authors' addresses: Z. Qi, J. Yao, C. Zhang, M. Yu, and Z. Yang, the School of Software, and the Shanghai Key Laboratory of Scalable Computing and Systems, Shanghai Jiao Tong University, 800 Dongchuan Road, Shanghai, 200240, China; emails: \{qizhwei, jianguo.yao, kevin_zhang, boats0425\}@sjtu.edu.cn; M. Yu (current address), Cylab, Carnegie Mellon University, 5000 Forbes Ave, Pittsburgh, PA 15213, USA; email: superymk@cmu.edu; H. Guan, the Shanghai Key Laboratory of Scalable Computing and Systems, and the Department of Computer Science, Shanghai Jiao Tong University, 800 Dongchuan Road, Shanghai, 200240, China; email: hbguan@sjtu.edu.cn.

Permission to make digital or hard copies of part or all of this work for personal or classroom use is granted without fee provided that copies are not made or distributed for profit or commercial advantage and that copies show this notice on the first page or initial screen of a display along with the full citation. Copyrights for components of this work owned by others than ACM must be honored. Abstracting with credit is permitted. To copy otherwise, to republish, to post on servers, to redistribute to lists, or to use any component of this work in other works requires prior specific permission and/or a fee. Permissions may be requested from Publications Dept., ACM, Inc., 2 Penn Plaza, Suite 701, New York, NY 10121-0701 USA, fax +1 (212) 869-0481, or permissions@acm.org.

(c) 2014 ACM 1544-3566/2014/06-ART17 $\$ 15.00$

DOI: http://dx.doi.org/10.1145/2632216 


\section{INTRODUCTION}

Cloud computing permits a significant reduction in the cost of capital and equipment maintenance by allowing users to host their software in the cloud data centers under a simple pay-as-you-go service. Cloud data centers are constructed around virtualization technology. Therefore, virtualization technology has a significant impact on how resources are used and managed in cloud services. Several virtualization solutions (e.g., VMware products, Xen [Barham et al. 2003], VirtualBox) are getting more and more mature for constructing huge cloud computing centers. As an open source platform, which is popular in the academic world, Xen is a pioneer in paravirtualization technology. Many prior research works are accomplished on it. VMware is another powerful commercial virtual machine (VM). It provides the user with a robust virtualization system that includes $3 \mathrm{D}$ acceleration.

Virtualization technology has been successfully applied to a variety of devices; however, graphics processing unit (GPU) virtualization is still at an early stage but has developed dramatically over the past few years. Due to the powerful performance of floating-point arithmetic as well as cost-efficiency, GPU virtualization has been widely studied, especially in the domain of high performance computing (HPC). Several research works [Ravi et al. 2011; Phull et al. 2012; Gupta et al. 2009; Shi et al. 2009; Duato et al. 2010] leverage GPU virtualization for general-purpose computing on graphics processing units (GPGPUs). Virtualized GPGPU systems intercept vendor-specific libraries such as Nvidia CUDA, OpenCL, and AMD Accelerated Parallel Processing and redirect the requests to the hypervisor where they are actually processed. Once the requests are served, the results are sent back to the corresponding guest operating system (OS). It is a vital purpose that GPU resources in these virtualized GPGPU systems are efficiently shared, as doing so can bring much improvement to cloud data centers. It highlights that HPC applications running on systems such as GViM [Gupta et al. 2009], vCUDA [Shi et al. 2009], rCUDA [Duato et al. 2010] have reached almost the same performance level as those running in native, nonvirtualized environments.

In addition to GPGPU, the other main application scenarios GPUs includes graphical processing such as gaming or 3D rendering. Techniques of GPU virtualization for graphics processing such as VGA-passthrough [Ng et al. 2009] and GPU paravirtualization [Lagar-Cavilla et al. 2007; Dowty and Sugerman 2009] are reaching their maturity. We conducted an experiment to evaluate the performance of applications in VMware. We used Windows 7 as both the guest and host OS, and 3DMark06 as the benchmark to test the performance. As a result, VMware Player 4.0 achieves $95.6 \%$ of the native performance, whereas VMware Player 3.0 only achieves $52.4 \%$. Due to these technological advances, there is an ever-increasing number of data centers dedicated GPU computing tasks such as cloud gaming and video rendering. For instance, in cloud gaming, the platform renders games remotely and streams the result over the network so that clients can play high-end games without owning the latest hardware. Many cloud gaming service providers such as OnLive ${ }^{1}$ have become publicly known in the past 4 years. OnLive is currently partnering with more than 90 publishers and servicing almost 300 games online.

However, how a graphics card is shared among games running on top of the VMs is not well studied. Resource sharing in existing virtualization solutions is often poor. For example, today, most cloud gaming service providers run multiple instances of a game, entirely allocating one GPU for each instance, although it may require very little or even no GPU computation resource. Proprietary motherboards are also used to host more GPU adapters on a single machine. On the other hand, game developers heavily

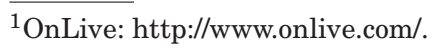


optimize their products to meet the capacity of midrange hardware. Hence, such ways of deploying cloud game servers cause a waste of hardware resources.

This article proposes VGRIS, a scheduling framework for virtualized GPU resource isolation and scheduling. VGRIS transparently enables different VMs composed of VMware and VirtualBox in the cloud to efficiently share a single GPU. Leveraging GPU paravirtualization technology, VGRIS is a lightweight resource scheduler in the host. A major challenge in resources management on a GPU is related to the graphics processing that is executed in an asynchronous and nonpreemptive way. Specifically, VGRIS adopts a library application programming interface (API) interception to take care of the underlying scheduling. In contrast with GViM, vCUDA, and rCUDA, VGRIS intercepts the library of graphics processing instead of the one of GPU programming. A major benefit of the library API interception is that we only need to modify a few binaries within the intercepted library. No other part of the software stack on top of the physical machine needs to be changed to embrace VGRIS. Moreover, VGRIS does not need any source code or design information on the library to perform such modification.

As an extension of our prior work [Yu et al. 2013], the whole framework is abstracted as a platform where GPU resources are scheduled. We extend the framework with a set of API to make the framework itself host a variety of scheduling algorithms. Such API not only allows assigning specific schedulers to the framework but also provides additional information including FPS, frame latency, and so forth, of the games running in VMs. Based on the API, VGRIS can support many scheduling algorithms that are designed and implemented in different ways. VGRIS is also able to select among all of the algorithms in a simple and fast way.

In our previous work [Yu et al. 2013], we only used VMware to evaluate VGRIS. Compared to the old version, we extend the framework to adapt to an additional VM of VirtualBox that also supports 3D acceleration. The heterogeneous virtualization platform makes it hard for the old implementation to schedule GPU resources among different VMs. To eliminate the problem, we improve the API to enable VGRIS to scheduling GPU resources even on heterogeneous platforms.

Currently, we implement three scheduling algorithms by the API within VGRIS, just as we did in our previous storage scheduling system [Wang and Merchant 2007]. They address the trade-off between a service-level agreement (SLA) and the throughput. More precisely, SLA-aware scheduling strives to achieve SLA requirements for each VM, which can benefit cloud gaming platforms. However, the GPU may not be fully utilized under this scheduling policy. Therefore, another policy, namely proportional-share scheduling, allocates the GPU resources to each VM in proportion to its given weight. In turn, this can greatly benefit job prioritization when rendering frames as well as the total throughput for the same cost as an SLA. Furthermore, VGRIS introduces a hybrid scheduling that guarantees minimum resources for the SLA while proportionally sharing the exceeding resources among all VMs. This third policy features better resource utilization than SLA-aware scheduling and prevents starvation that may occur with a proportional share. In particular, more advanced scheduling algorithms can be implemented within VGRIS by the proposed API in the future.

The workloads in our previous work included 3DMark05 and two games. However, these workloads are not appropriate for cloud gaming scenarios. Typical cloud gaming service providers always give access to real-world games for users and tend to run multiple games concurrently on a single server. To prove that VGRIS is practical and feasible under such circumstances, we conducted the experiments by running three real-world games-DiRT 3, Farcry 2, and Starcraft 2-both on VMware and heterogeneous platforms. The experimental results show that VGRIS can effectively schedule GPU resources for real-world games even on heterogeneous platforms. The results also show that all the three scheduling policies satisfy their design goals under 
various workloads. For example, when applying the SLA-aware scheduling, the average frames per second (FPS) of the workloads increases by $65 \%$. The percentage of frames with excessive latency drops to $0.20 \%$. In the meantime, the performance overhead incurred by VGRIS is limited to $3.59 \%$.

The contributions of this article are summarized as follows:

-We propose a GPU scheduling framework based on the GPU paravirtualization architecture, which can be applied to servers for various GPU computing tasks for efficient resource management. Benefiting from the library API interception, VGRIS is lightweight and does not require any source code changes in the guest OS, the guest game, or the host graphic drivers.

-A set of API is provided to make VGRIS able to support a variety of scheduling algorithms. All of these algorithms can be replaced with another one in a very simple and fast way. Benefiting from the API, VGRIS can leverage more advanced and powerful scheduling algorithms to schedule GPU resources for cloud games.

-We implement three scheduling policies by the API for the proposed framework, depending on different typical performance requirements: high performance of SLAs, proportional resource sharing, and performance and fairness trade-offs. The design and implementation of the three algorithms prove the usefulness of the API within the framework.

-We implement VGRIS and conduct several experiments by running real games and benchmark programs on it to demonstrate the effectiveness of our framework and of its scheduling policies. The overhead of the framework is limited to $3.59 \%$.

-We evaluate VGRIS on heterogeneous virtualization platforms consisting of VMware and VirtualBox. The results show that VGRIS can schedule GPU resources on more than one virtualization platform at the same time.

The rest of the article is organized as follows. Section 2 describes some motivating experiments that highlight the poor performance of the default GPU scheduling mechanism. Sections 3 and 4 respectively introduce the design and implementation of the VGRIS framework as well as three scheduling policies integrated into VGRIS. Section 5 presents experimental results based on the use of real games and benchmark programs. Section 6 discusses some related work, and Section 7 concludes this article.

\section{MOTIVATION}

This section mainly describes some motivating experiments that underscore the poor performance and low utilization of the default GPU resource scheduling. We also present a detailed analysis of the problem. We conducted the experiments on a machine with a midrange central processing unit (CPU) and an ATI HD6750 graphics card. Before analyzing the poor performance and low utilization when running multiple VMs on a single graphics card, we first briefly describe the standard 3D rendering and programming model and then discuss how the original graphics library schedules the GPU resources.

\subsection{GPU Computational Model}

Basically, GPU processing as well as CPU computation determines FPS rate. Figure 1 displays the GPU computation for various applications (e.g., gaming, rendering, stream processing), which are usually processed in an infinite loop [Owens et al. 2008]. Each loop determines exactly one frame.

First, UploadComputeKernel uploads the computation program to the GPU, and DeclareThreadGrid specifies the number of threads to be used for the computation. After the initial setup, each iteration of the loop performs some tasks, such as arithmetic calculating, drawing a frame, and rendering. There are three stages in each iteration: (1) some GPU data are prepared for CPU computation (e.g., calculating 


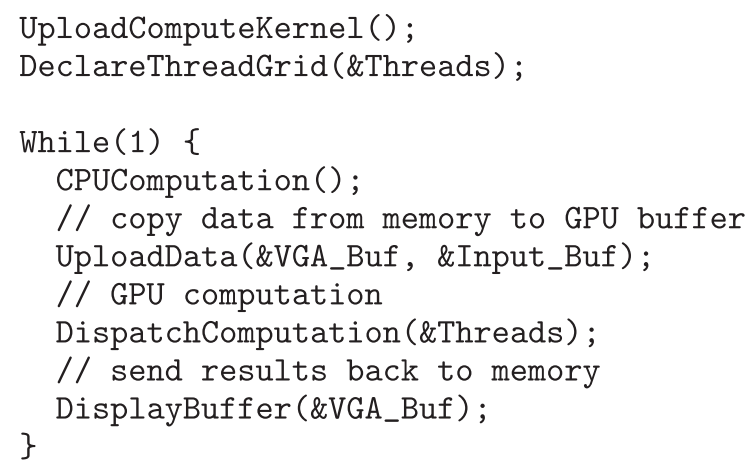

Fig. 1. GPU computation model.

Table I. Performance of Games Running Individually on iCore7 2600K + HD6750

\begin{tabular}{|l||c|c|c|c|c|c|}
\hline \multirow{2}{*}{ Game } & \multicolumn{3}{c|}{ Native Performance } & \multicolumn{3}{c|}{ Performance in VMware } \\
\cline { 2 - 7 } & FPS & GPU Usage & CPU Usage & FPS & GPU Usage & CPU Usage \\
\hline \hline DiRT 3 & 68.61 & $63.92 \%$ & $43.24 \%$ & 50.92 & $65.80 \%$ & $16.79 \%$ \\
Starcraft 2 & 67.58 & $58.07 \%$ & $47.74 \%$ & 53.16 & $76.62 \%$ & $18.64 \%$ \\
Farcry 2 & 90.42 & $56.52 \%$ & $61.36 \%$ & 79.88 & $82.44 \%$ & $26.66 \%$ \\
\hline
\end{tabular}

objects in the upcoming frame according to the game logic); (2) the data are uploaded to the GPU buffer, such that it can schedule its threads to perform the computation (e.g., rendering the frame from the buffer contents); and (3) the frame is rendered in the VGA buffer and then output on the screen, as done by DisplayBuffer in Figure 1. The detailed API depends on the graphics library, such as glutSwapBuffers from OpenGL and Present from Direct3D.

\subsection{Inefficiency of the Default GPU Sharing}

We now present some experiments to highlight the potential throughput improvements in a shared GPU environment while guaranteeing the SLA of each workload. We start by evaluating the performance of the individual workloads on the platform with Windows 7 as the host OS. We chose three popular games, DiRT 3, Farcry 2, and Starcraft 2, as the experimental workloads. Table I displays the performance results, in which GPU usage is calculated based on hardware counters. Usually, cloud gaming requires an FPS rate in the range of 30 to 60 for a smooth user experience. A lower rate would render the game unplayable, whereas a higher one would not make any difference to the human eye. As we can see from the figure, all of the workloads are able to provide a smooth user experience. However, running these workloads individually results in a waste both GPU and CPU resources. For instance, when running directly on the host OS, DiRT 3 only occupies about $63.92 \%$ of GPU utilization and $43.24 \%$ of CPU utilization. The rest of the GPU and CPU resources are sufficient enough to play another game, even on our midrange ATI HD6750 graphics card. Since cloud gaming service providers like OnLive upgrade their CPUs GPUs every 6 months [Joystiq 2009], running these games with dedicated GPUs will inevitably cause an unnecessary low GPU utilization and a waste of resources.

Table I also indicates the performance overhead of VMware. When running inside VMware, all three workloads have lower FPS rates and CPU usages but have higher GPU usages. The intervention of VMware incurs performance overhead, shown from the FPS rate results. Meanwhile, running the workloads inside VMware also results in extra GPU computation. The FPS overheads for the three workloads are $25.78 \%$, $21.34 \%$, and $11.66 \%$, respectively. The overhead incurred to GPU computation is within 


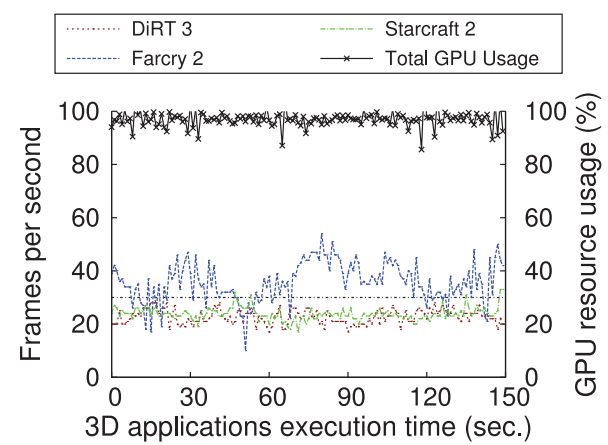

(a)

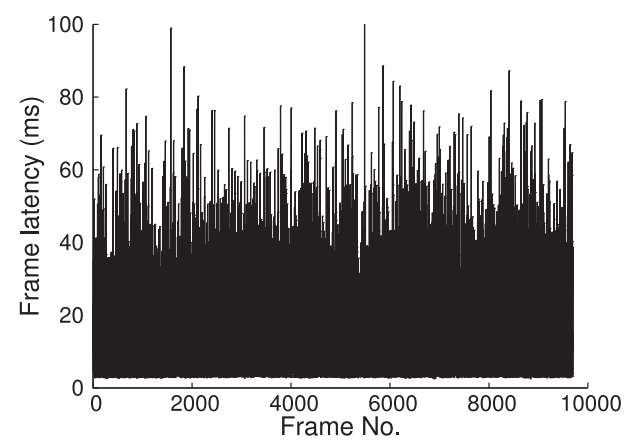

(b)

Fig. 2. Poor performance of the default scheduling mechanism under heavy contention. (a) FPS of the three workloads: DiRT 3, Farcry 2, and Starcraft 2. (b) Frame latency of Starcraft 2.

the range of $2.94 \%$ to $45.86 \%$. In particular, all three workloads inside VMware run at an FPS rate higher than 30, which concludes that the GPU virtualization technology of VMware is mature enough for games to provide a smooth user experience.

Based on this observation, Figure 2 shows the performance results of running multiple-game VMs on a single ATI HD6750 graphics card. The experiment involves three popular games: DiRT 3, Farcry 2, and Starcraft 2. Each workload concurrently runs in a separate VM of VMware where the guest OS is Windows 7 with Direct3D.

As shown from the figure, Starcraft 2 has an average FPS rate of 24, and DiRT 3 slows down to an average value around 23. Compared to their original performance under the same game configuration, their FPS is reduced dramatically. Despite the overhead incurred by the VM, the heavy contention of the GPU resources significantly influences the FPS rates of the workloads. From the figure, we can also see that the GPU resources are almost fully utilized. However, the FPS rates of DiRT 3 and Starcraft 2 are too low to provide a smooth gaming experience, which is mainly caused by the inefficiency of the default GPU sharing mechanism. In addition to the FPS rate, the user experience also depends on the frame latency that defines the time cost of one frame. Figure 2(b) illustrates the corresponding frame latency of Starcraft 2. As we can observe, this game suffers a high latency when competing with other games for GPU resources. More than $12.78 \%$ of the frames are beyond $34 \mathrm{~ms}$, and $1.26 \%$ frames are even beyond $60 \mathrm{~ms}$; the maximum latency almost reaches $100 \mathrm{~ms}$. This is a serious issue, as the larger the frame latency, the rougher the player's experience.

In terms of frame rate variance, the three VMs have values of 7.39, 55.97, and 5.83, respectively. All of the FPS rates fluctuate, which is consistent with the feature of realworld games, especially Farcry 2. This is because Farcry 2 is a first-person shoot game, and its FPS rates vary dramatically when the game is running.

The most likely reason for the default poor resource scheduling mechanism is the asynchronous and nonpreemptive nature of the GPU process. For instance, the default GPU scheduling mechanism in Direct3D runtime library tends to allocate resources on a first-come first-served manner, which results in an excessive FPS rate for low-end games and an unplayable FPS rate for GPU-demanding games when they are running concurrently in separate VMs. Graphics APIs also typically work in an asynchronous way to maximize hardware performance. APIs such as DisplayBuffer in Figure 1 immediately return when they issue and submit a rendering command to the GPU. The GPU maintains a command buffer for upcoming user space requests. Therefore, if the underlying command buffer is full, the $3 \mathrm{D}$ application has to be blocked for some time. 


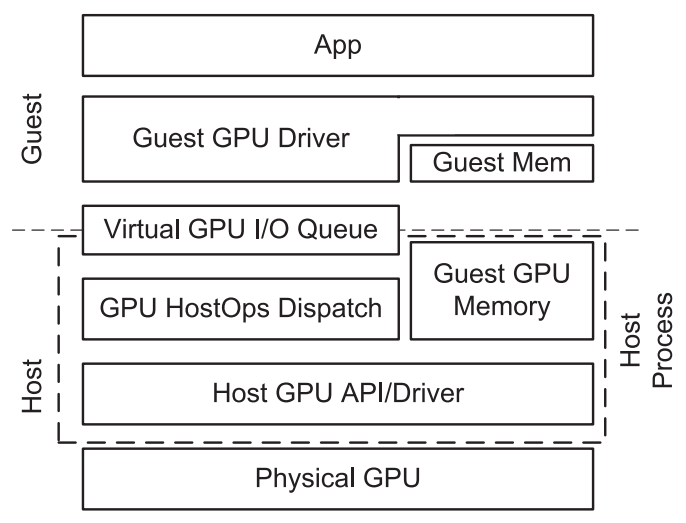

Fig. 3. GPU paravirtualization architecture.

For instance, in a typical 3D application development, every 3D application creates a unique Direct3D device to represent its own graphics context. The Direct3D calls issued by an application are usually converted into device-independent commands, batched in a command queue within the application context. When the command queue is full or at an appropriate time, the Direct3D runtime submits the current device command queue to the underlying GPU driver. The driver stores the coming queue into its local command buffer for the GPU cores to process it asynchronously. Some commands are kept by the Direct3D runtime until the available room is found in the command buffer on the driver side. Thus, if two or more 3D applications run concurrently on a single graphics card, a resource contention inevitably occurs. If one 3D application runs a little fast and frequently submits its command queue to the underlying layer, it probably obtains more GPU resources. At the same time, another 3D application might suffer severe starvation, causing a low FPS rate as it is running. Besides, it is important to note that a 3D application needs to recreate GPU resources after its windows are updated. Hence, it is common that only one GPU-accelerated 3D application occupies the whole GPU for a period of time regardless of the number of cores or threads in the GPU.

\section{VGRIS SYSTEM ARCHITECTURE}

\subsection{GPU Paravirtualization and VGRIS Architecture}

Paravirtualization provides VMs with a software interface that is different from its underlying hardware counterpart. This interface significantly reduces the overhead of operations that are substantially more difficult to run in a virtual environment. The guest OS must be explicitly ported to exploit the new interfaces for better performance. In the case of a commercial OS that cannot be modified, this is often achieved by paravirtualization-aware device drivers. Due to the complexity of the GPU device drivers, it is only recently that hypervisors supporting GPU paravirtualization have achieved near-native efficiency.

Figure 3 shows the typical GPU paravirtualization architecture for type 2 (hosted) hypervisors [Dowty and Sugerman 2009]. Typically, a GPU rendering task issued by a guest OS application is executed as follows. After the guest application invokes a standard GPU rendering API, the guest GPU computation library (e.g., OpenGL, Direct3D) prepares the corresponding GPU buffer contents in the main memory and issues the GPU command packets. These packets are pushed into the virtual GPU I/O queue, which are subsequently processed by the Host0ps Dispatch in the host. Finally, this last layer sends the commands to the device driver in an asynchronous fashion. 


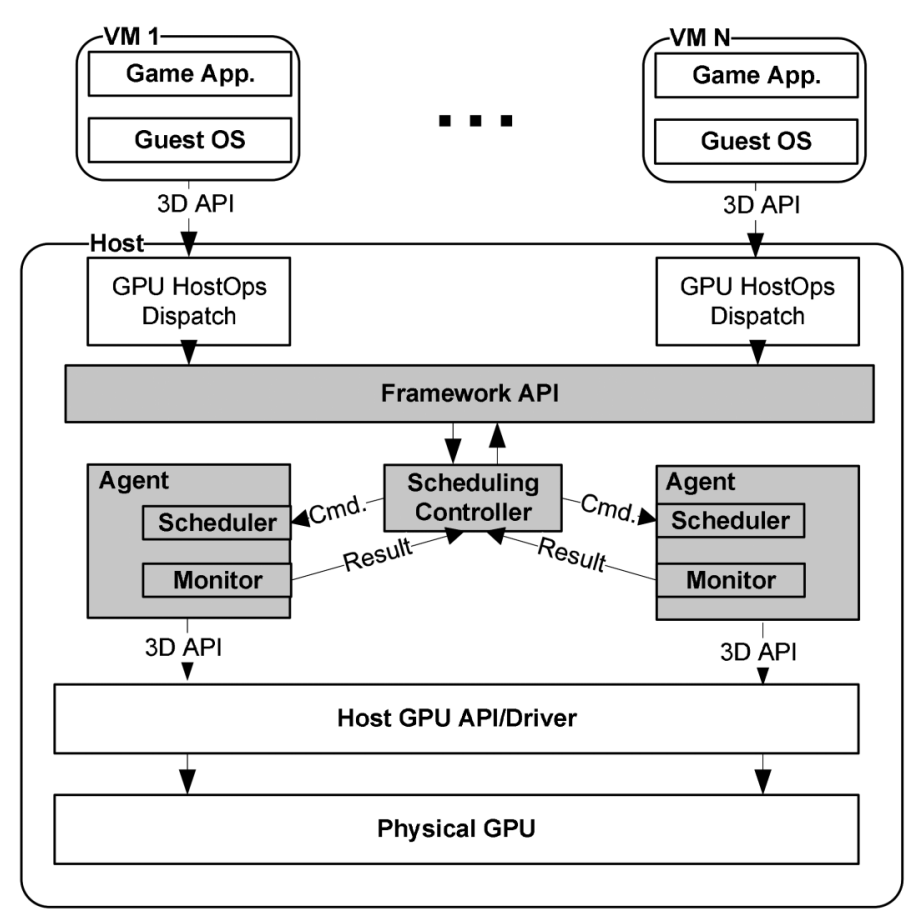

Fig. 4. VGRIS architecture.

The buffer contents of the guest OS memory are transferred into the GPU buffer using direct memory access (DMA) through this process.

Figure 4 represents the VGRIS architecture within the paravirtualization framework shown in Figure 3, where modules introduced by VGRIS are highlighted in grey. These modules are all inside the host. There is one agent per VM, which schedules GPU computation tasks and monitors the performance. In addition, there is a centralized scheduling controller that serves two purposes. First, it receives the commands from the administrator to decide which scheduling algorithm is used. Second, under the hybrid scheduling policy, it automatically selects between the SLA-aware and the proportional-share policy based on the performance feedback received from all agents. In addition, the content and the frequency of the performance report from each agent are specified by the central controller. The scheduling algorithm does not require any feedback. In the implementation of the prototype, each agent simply intercepts the Direct3D API invocations from the GPU HostOps Dispatch for rescheduling. Its performance monitoring function utilizes the GPU performance instrumentation methods. The centralized scheduling server is implemented as an independent process. In addition, VGRIS provides API for scheduling algorithms to leverage. The API can make VGRIS host a variety of scheduling algorithms without modifying VGRIS itself. The detailed description is provided in the next section.

\subsection{API and Algorithms Overview}

Based on the prior framework introduced in Yu et al. [2013], we abstract the functions in the form of API to provide interfaces so that a variety of scheduling algorithms can be implemented within the framework without any modification to the framework. The followings are the detailed descriptions of the main functions: 
(1) StartVGRIS sets a startup of all modules of the framework. It first does all of the initialization, including library interception and process hooking. Then it starts up the scheduler controller and the agent for each running game.

(2) PauseVGRIS pauses the framework temporarily. After this function is invoked, the framework will no longer schedule GPU resources for the VMs while the games run in their original FPS rates. The framework can be made to work again by invoking ResumeVGRIS.

(3) ResumeVGRIS resumes the framework if it is paused by PauseVGRIS.

(4) EndVGRIS terminates all modules of the framework and does the cleanup work for VGRIS.

(5) AddProcess adds the process in the framework. The name and ID of the process will be added to the application list maintained by VGRIS. Then, it can be scheduled by the framework. Leveraging this interface, VGRIS can schedule GPU resources on heterogeneous virtualization platforms composed of VMware and VirtualBox.

(6) RemoveProcess removes the process from the application list of the framework. After this interface is invoked, the process will no longer be scheduled by the framework.

(7) AddHookFunc assigns a function to be hooked to the given process. Every process in the framework has a function list that consists of the functions to be hooked by the framework. This interface adds the specified function to the list and then notifies VGRIS to hook it. The process must be in the application list of the framework; otherwise, this interface will return an error to the caller.

(8) RemoveHookFunc removes the function that is currently hooked by the framework from the function list of the given process.

(9) AddScheduler adds the specific scheduling function to the scheduler list maintained by VGRIS. Meanwhile, VGRIS assigns an ID to the scheduler and returns the ID to the caller. A scheduler implements the detailed procedure of how to schedule GPU resources. After it is added to the framework, it will be invoked by the framework in each iteration of the running games to do the scheduling.

(10) RemoveScheduler removes the scheduler from the scheduler list according to the given ID.

(11) ChangeScheduler changes the current scheduler to the next one in the scheduler list in a round robin manner. If the scheduler ID is given as the parameter, VGRIS will change to the specified scheduler if it exists in the scheduler list.

(12) GetInfo collects the information about the running game from the monitor. Since each hooked process has a monitor, this function only gets the information of the game VM on which the monitor resides. A parameter is used to return the type of information. Currently, the information includes FPS, frame latency, CPU usage, GPU usage, scheduler name, process name, and function name.

Figure 5 demonstrates an example of using the aforementioned API. In the example, AddProcess and AddHookFunc are first called to add the processes and functions that will be hooked into the lists of the framework. AddScheduler is used to add the specified schedulers. Then, ChangeScheduler is called to make the framework leverage the scheduler, SpecifiedScheduler2 in this case, to schedule GPU resources for the VMs. After StartVGRIS is invoked, the specified scheduler begins to work. During scheduling, some processes and functions can be removed by RemoveHookFunc and RemoveProcess. ChangeScheduler can be used to replace the current scheduler with another one existing in the scheduler list of the framework. Finally, EndVGRIS can be used to terminate the scheduling. 


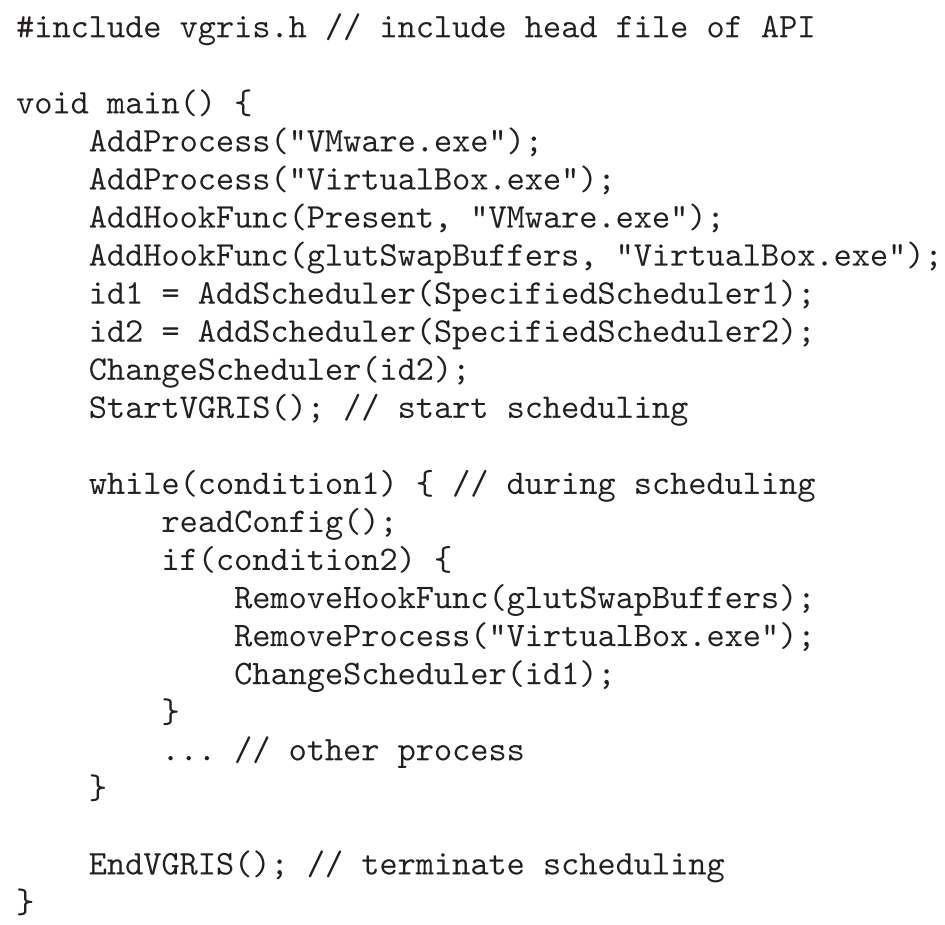

Fig. 5. An example of using the proposed API.

Using this API, we designed and implemented three representative scheduling algorithms for different optimization goals. In the future, more advanced scheduling algorithms will be implemented and integrated into VGRIS by this API. The algorithms are briefly described as follows:

-SLA-aware scheduling allocates just enough GPU resources to each VM to fulfill the SLA requirement (e.g., 30 FPS in most cases). Note that the GPU resources may be not fully used under this policy.

-Proportional-share scheduling allocates all GPU resources to all the running VMs in proportion to their weights, as assigned by the administrator.

- Hybrid scheduling with a compromise mixes the preceding two schemes. It first allocates a minimal amount of resources to each VM such that the SLA requirement is satisfied. Then extra resources are then proportionally allocated between all VMs to maximize the GPU utilization.

\section{VGRIS SYSTEM IMPLEMENTATION}

Before delving into a detailed implementation of VGRIS, we provide an overview of some challenges that resulted in the current implementation of VGRIS.

\subsection{Challenges for VGRIS and Discussions}

During the implementation of the VGRIS prototype, we faced several challenges. Next, we discuss those challenges and the corresponding technologies leveraged to overcome them.

Platform selection challenge. The first implementation problem relates to the choice of virtualization platform to implement VGRIS. Several virtualization solutions are 
Table II. Performance Comparisons between VMware and VirtualBox

\begin{tabular}{|l||c|c|}
\hline Workloads & FPS Rate in VMware & FPS Rate in VirtualBox \\
\hline \hline PostProcess & 639 & 125 \\
Instancing & 797 & 258 \\
LocalDeformablePRT & 496 & 137 \\
ShadowVolume & 536 & 211 \\
StateManager & 365 & 156 \\
\hline
\end{tabular}

available: Xen is a type 1 hypervisor, widely used in the academic community, whereas VMware and VirtualBox are representative type 2 hypervisors that can provide 3D acceleration. We chose a type 2 hypervisor and made use of the corresponding paravirtualization technology to allow the games in the VMs to invoke the graphics libraries of the host OS. The reasons are given as follows. Graphics libraries are required by games to render scenes onto the screen, but they are so powerful, large, and sophisticated that it is not practical to integrate them in a hypervisor. In addition, as explained in Section 1, the most versions of VMware and VirtualBox both provide 3D graphics libraries for the guest OS, which means that 3D applications running inside the VMs can get 3D acceleration.

In this article, VGRIS is implemented both on VMware and VirtualBox with the support of the proposed API. However, we mainly focus on VMware due to the performance and 3D feature compatibility. Table II shows the performance comparisons of VMware and VirtualBox. All of the workloads are from DirectX SDK. They all run with the same configurations and settings. From the table, we can see that VMware has a significant performance advantage over VirtualBox when hosting 3D applications in their VMs. Running PostProcess makes the most performance gap in that the 3D performance of VMware is five times faster than that of VirtualBox. In our analysis, VirtualBox requires translating the graphics library invocation from Direct3D API to OpenGL API when it runs 3D applications using the Direct3D graphics library. For instance, when PostProcess invokes Present, a rendering function of the Direct3D library, the hypervisor of VirtualBox receives the request and then translates it to glutSwapBuffers, the corresponding rendering function in OpenGL. Finally, the translated request is sent to the host OS. However, VMware does not perform such a translation operation when hosting 3D applications. As a consequence, VMware has a better performance than VirtualBox in terms of the games implemented using the Direct3D library. Therefore, we make more efforts on integrating VMware as the virtualization platform in VGRIS. Moreover, it is observed that VirtualBox does not support all features of 3D libraries, especially the advanced and complicated ones. For instance, in our experiments, VirtualBox is not compatible with those $3 \mathrm{D}$ games that require Shader $3.0 .^{2}$ Taking these reasons into account, we decide to run real-world games in VMs of VMware and benchmarks from DirectX SDK in the VMs of VirtualBox in the evaluation section.

Challenge of library interception. As shown in Figure 4, VGRIS resides on the host OS and intercepts the Direct3D library calls from the guest OS. Benefiting from the library interception, VGRIS treats the VMs as black boxes allowing the prototype to be implemented without modifying the graphics libraries of the guest and the host OS, or the drivers of the graphics card. Thus, the library interception becomes the key issue of the prototype implementation.

One way to intercept the Direct3D API on Windows 7 is to modify the hypervisor of the VMs. Hypervisors supporting of paravirtualization technology redirect the graphics API calls from 3D applications directly to the corresponding API on the host.

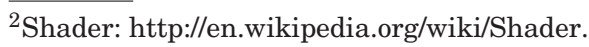




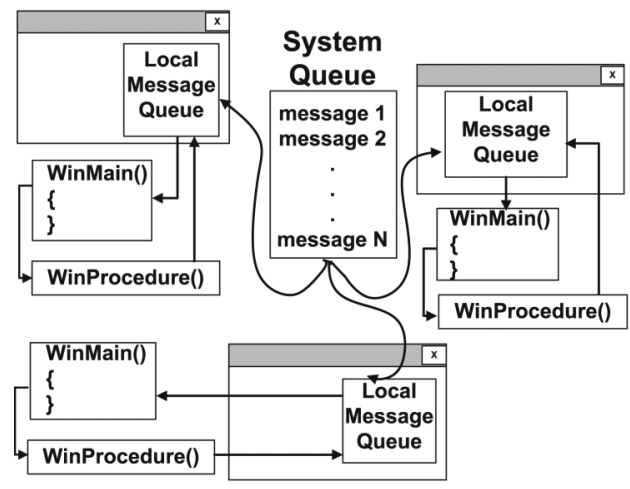

(a)

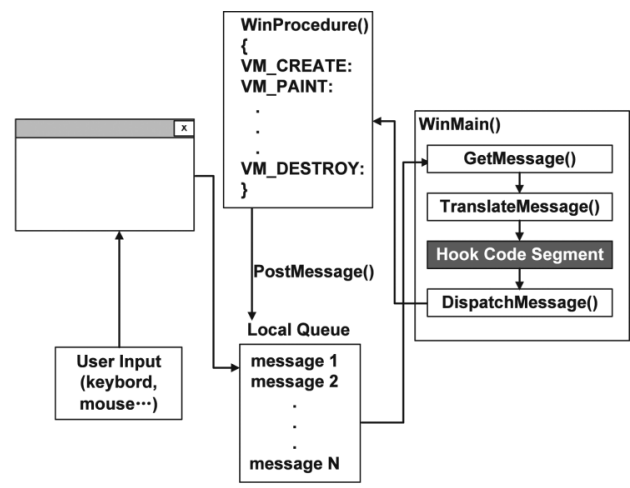

(b)

Fig. 6. Default message loop model and hook technology under Windows. (a) Default message loop procedure. (b) Message loop procedure after hook.

We can modify the redirection procedure and insert the codes of the three scheduling policies. Note that a different virtualization solution could be selected if another hypervisor displays a better 3D acceleration. However, under such circumstances, the whole architecture should be totally reimplemented, which renders VGRIS inflexible when choosing a different VM.

Another way to implement library interception is to leverage the message processing mechanism of the Windows OS. The technology is called hook, and it can monitor the message loop of applications running under Windows. A detailed introduction to the hook mechanism is provided in Section 4.2. Overall, in the hook technology, an application is able to intercept a particular type of message and specify a function to deal with it before it is sent to the Windows applications. Hence, the greatest advantage of leveraging hook as the graphics API interceptor is that the hypervisor, the host OS, and the underlying device driver do not need to be modified when employing VGRIS, thus rendering it much more flexible when switching to a different virtualization solution.

\subsection{Hook and Library Interception}

A hook is a Windows technology through which an application can intercept events, such as messages, mouse actions, and keystrokes. In fact, a hook is a piece of code segment to process events. It intercepts a Windows application using a system call, SetWindowsHookEx. An important parameter of the system call specifies which event is to be intercepted. Another interesting parameter is an entry to the hook procedure that is invoked just before the particular type of event is handled by the default procedure function of the hooked Windows application. The counterpart of SetWindowsHookEx is UnHookWindowHookEx, which removes the code segment used by hook from the hooked Windows application. Once UnHookWindowHookEx is invoked, the specified hook procedure is no longer invoked when the event is sent to the hooked Windows application.

Figure 6(a) shows the default message loop model of a Windows OS. The system maintains a global message queue that collects messages to update the Windows applications. For instance, actions such as resizing a window, clicking the mouse, or pressing the keyboard generate a message enqueued by the OS. The default procedure of the Windows application can also send messages to itself or other Windows applications by invoking PostMessage, but the message is first enqueued into the global queue. Then, the OS dispatches the messages in the queue one by one to the corresponding Windows applications. The Windows application also keeps a local queue to store the messages 


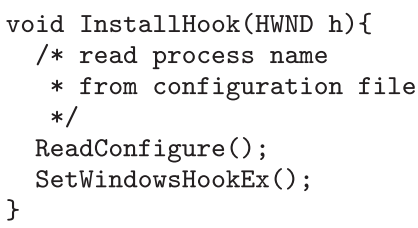

(a)

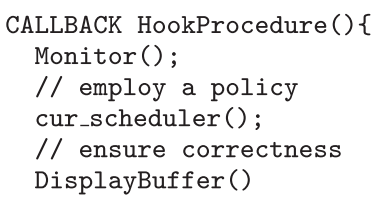

(b)

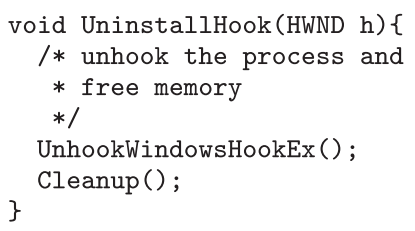

(c)

Fig. 7. Implementation of VGRIS by hook. (a) InstallHook. (b) HookProcedure. (c) UninstallHook.

and has a loop to process the messages from its local queue, as shown in Figure 6(a). The loop will not exit until a quit message is sent to the application. In each iteration of the loop, the application picks a message from its local message queue and decides to use the callback function to process it. The hook technology just interposes the loop by anticipating the message before the default loop processing mechanism of the application handles it. Figure 6(b) depicts the procedure. After hooking, a piece of code is inserted into the loop, which first gets the messages from the local queue and then translates them into a form where they can be identified. If the message is specified by SetWindowsHookEx, it will be then processed by the hook procedure; otherwise, the message is processed by the default procedure of the application. The hook technology dramatically simplifies the implementation of VGRIS and also makes it applicable to all Windows platforms.

With the support of the hook technology in the Windows OS, the prototype first invokes SetWindowsHookEx in the InstallHook function, as shown in Figure 7(a). InstallHook hooks the process by the given name or ID. Then, it passes the address of both the DisplayBuffer from the graphics library and the hook procedure to SetWindowsHookEx. We choose to intercept the rendering function call simply because it performs rendering jobs in each loop of the GPU computation model. It is also possible to extend the scheduling framework in a simple and fast manner by specifying more messages that are to be monitored.

After all of the preparation is complete, VGRIS is able to monitor all of the VMs running under Windows. Once a VM invokes a DisplayBuffer call to render its frames, the hook function, HookProcedure, is called. The implementation is shown in Figure 7(b). As shown in the figure, a monitor and scheduler run in theHookProcedure of each hooked process, which corresponds to Figure 4. Monitor collects necessary information such as the current FPS from the VM. cur_scheduler is the function pointer that points to a specific scheduling policy. It can be replaced with different scheduling policies. In the next section, we present the three policies that can be invoked. After the scheduling, DisplayBuffer is invoked again to ensure that the original frame rendering procedure is properly applied.

Finally, the prototype invokes the UninstallHook function to clean up, as shown in Figure 7(c). The function unhooks the VM process and then frees the memory allocated by VGRIS.

\subsection{Implementation of VGRIS API}

The main interfaces of the framework are listed in Section 3.2. This section mainly discusses the implementation of these interfaces.

Start VGRIS and End VGRIS are two interfaces that correspond to start and terminate the framework. The former one invokes InstallHook and intercepts all existing functions in the function lists of all the processes maintained by VGRIS. Thus, the HookProcedure, including the monitor and scheduler, as shown in Figure 7(b), is injected into the target process. The latter one reverses the procedure. It invokes UninstallHook and does the cleanup work. Pause VGRIS just invokes UninstallHook 


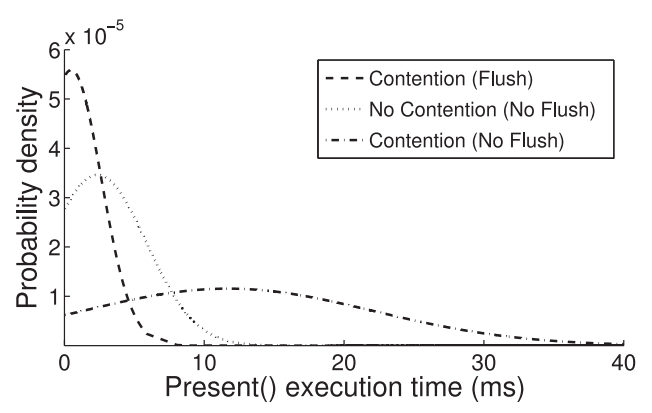

Fig. 8. Probability distribution of Present time cost.

for all functions of all the processes so that the GPU resources are temporarily not scheduled and games run in their original FPS rate. Resume VGRIS calls InstallHook again to resume the work of the framework.

AddProcess just adds the process name and ID to the application list maintained by the framework. RemoveProcess removes the process specified by the parameter from the list. AddHookFunc and RemoveHookFunc are implemented in a similar way.

AddScheduler adds the definite scheduler to the scheduler list of the framework. If the scheduler is the only one on the list, the framework will assign it to cur_scheduler. cur_scheduler is a variable with the type of function pointer that points to the current scheduler used by the framework. In this way, VGRIS can simply host a variety of scheduling algorithms without modifying any part of the framework. ChangeScheduler allows the framework to use another scheduler in the list in a round robin manner. RemoveScheduler removes the function pointer from the scheduler list. If the scheduler to be removed is being used by the framework, it will invoke ChangeScheduler to make the framework change to another scheduler.

GetInfo collects the current information about the running game from the monitor. It returns the FPS, frame latency, CPU usage, GPU usage, scheduler name, process name, and function name that are hooked according the given parameter of type. The FPS of a game is derived from the frame latency. As shown in Figure 1, each iteration determines exactly one frame. The frame latency can be calculated based on the CPU computation time of ComputeObjectsInFrame and DrawPrimitive, and the GPU computation time of DisplayBuffer. The CPU computation time can be simply measured. However, the GPU computation time can only be predicted.

In the experiments, we observe a variation in the computational time of Present, the API from Direct3D corresponding to DisplayBuffer. In fact, on heavy contention, the average execution time of Present raises from $2.37 \mathrm{~ms}$ to $11.70 \mathrm{~ms}$, as shown in Figure 8. This is due to the DirectX runtime batches that Direct3D commands for better efficiency. Hence, heavy contention increases the possibility of full command buffer, resulting in a less predictable execution time for Present. The Flush command can significantly mitigate the problem. Figure 8 shows that the average computation time of Present is reduced from $11.70 \mathrm{~ms}$ to $0.48 \mathrm{~ms}$ under heavy contention. The Flush command induces extra CPU computational cost. Since we mainly consider GPU-bounded $\mathrm{VMs}$, it is reasonable to spend a little extra CPU time for a more accurate prediction and therefore achieve a more stable latency for each frame. By inserting a Flush in each iteration, we can predict the GPU computation time more precisely.

\subsection{Scheduling Algorithms}

To prove the feasibility and flexibility of the current framework, we leverage the newly designed API to implement three scheduling algorithms. In the implementation of all 


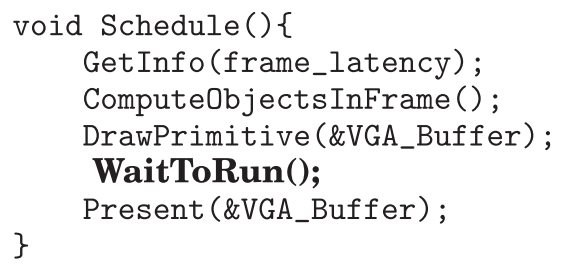

(a)

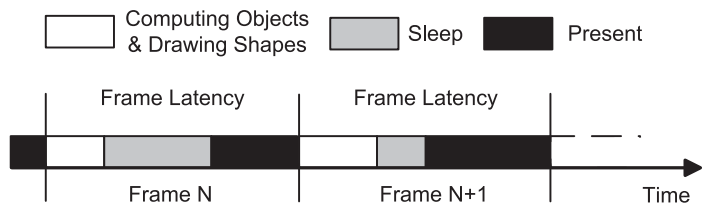

(b)

Fig. 9. SLA-aware and proportional-share scheduling. (a) Pseudocode of the scheduling algorithms. WaitToRun() maps to Sleep(calculated_sleep_time) when SLA-aware is the current scheduler. It maps to WaitForAvailableBudgets() when proportional-share is the current scheduler. (b) Frame latency of one frame in SLA-aware scheduling.

scheduling algorithms, the framework itself is not modified. With the support of the API, other scheduling algorithms are also applicable to the VGRIS architecture.

SLA-aware scheduling. From the SLA requirements in cloud gaming, service providers try to guarantee a minimum FPS and a maximum latency for a smooth user experience. As Figure 2(a) illustrates, the default GPU scheduling algorithm allocates resources on a first-come first-served strategy, even under heavy contention. As a result, less-GPU-demanding 3D applications may get more resources than necessary, whereas GPU-demanding ones cannot meet the SLA requirement. SLA-aware scheduling is designed to address this issue. It allocates just enough resources to each VM to guarantee its SLA. To achieve this goal, we slow down less-GPU-demanding games to provide extra resources for more GPU-demanding ones.

To stabilize the frame latency, we extend each frame by delaying its last call, Present. This is achieved via inserting a Sleep call before Present. Figure 9(a) shows the pseudocode of the scheduling algorithm. The Schedule function is assigned by AddScheduler and executes just before the Present, as shown in Figure 7(b). It is responsible for calculating the length of the delay, which should be equal to the desired latency minus the computation time of ComputeObjectsInFrame, DrawPrimitive, and Present altogether.

Proportional-share scheduling. SLA-aware scheduling strives to meet the SLA requirements, which may result in low resource utilization when there are an insufficient number of VMs. Proportional sharing is a scheduling mechanism that is very well suited for these application scenarios.

Figure 9(a) also shows the pseudocode of the proportional-share scheduling algorithm. When the scheduling algorithm is proportional share, WaitToRun in the figure maps to WaitForAvailableBudgets, which waits for the available GPU resources to be ready. Our proportional-share scheduling algorithm adopts the Posterior Enforcement Reservation policy used in TimeGraph [Kato et al. 2011b], which queues and dispatches GPU commands based on the task priorities. First each VM $i$ is assigned a share $s_{i}$ that represents the percentage of GPU resources that it can use for a period $t$. The shares of all VMs add up to one. The budget $e_{i}$ represents the amount of GPU time that the VM $i$ is entitled for its execution. This budget decreases following the amount of time consumed on the GPU and is replenished by at most $t s_{i}$ once every period $t$ as follows:

$$
e_{i}=\min \left(t s_{i}, e_{i}+t s_{i}\right) \text {. }
$$

The proportional-share scheduling dispatches the Present API invocation if the budget for the corresponding VM is greater than zero; otherwise, it is postponed. We set $t=$ $1 \mathrm{~ms}$ in our implementation, which is sufficiently small to prevent long lags.

Hybrid scheduling. SLA-aware scheduling may result in low GPU utilization with an insufficient number of VMs. On the other hand, proportional-share scheduling can 
maximize the utilization, but inappropriate weights can lead to some VM starving. Our hybrid scheduling mechanism combines the benefits of the two by automatically choosing the appropriate algorithm using the calculated parameters, as shown in Algorithm 1.

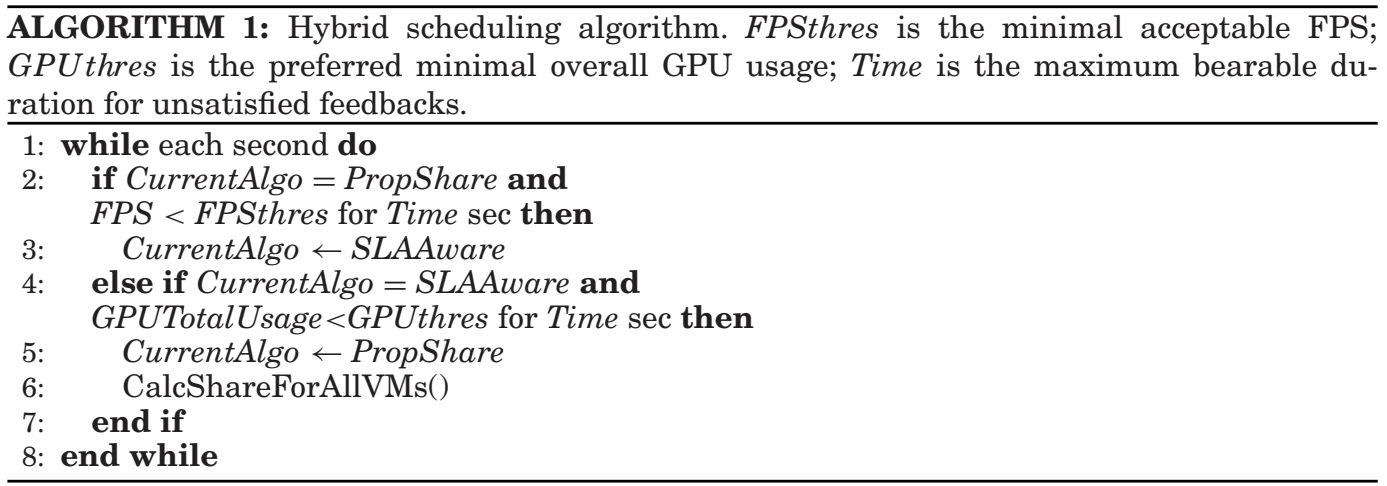

The hybrid scheduling algorithm leverages the API described earlier to collect the performance information from each VM. It then determines the appropriate scheduling algorithm for all VMs based on the collected information. When initialized, the hybrid scheduling algorithm retrieves the threshold values from the settings and employs proportional-share scheduling with a fair share as a default algorithm. During the runtime, any reported status below the criteria for the wait duration will lead to a change of the scheduling algorithm among all agents. For example, the administrator may indicate a wait duration of 5 seconds. If proportional-share scheduling is leveraged as the current scheduling algorithm for all VMs, hybrid scheduling uses the SLA-aware scheduling algorithm if and only if some VMs have a low FPS at the checkpoint. On the contrary, the proportional-share scheduling algorithm is selected if the current scheduling method is SLA-aware scheduling and the physical GPU usage is below a certain bound.

The hybrid scheduling algorithm needs to determine the proper share for each VM when switching to the proportional-share scheduling algorithm. The proportional share for the $i$-th VM $\left(s_{i}\right)$ is achieved as follows:

$$
s_{i}=u_{i}+\frac{\left(1-\sum_{i=1}^{n} u_{i}\right)}{n} .
$$

This formula approaches proportional sharing while guaranteeing the SLA for each VM. $u_{i}$ stands for the GPU usage of the $i$-th VM. It represents the minimum share of GPU resources needed when switching to proportional-share scheduling. $\left(1-\sum_{i=1}^{n} u_{i}\right) / n$ represents the fairness division of the abundant GPU resources for each VM. This fair division permits that every VM gets more GPU resources than required to fulfill the SLA requirement in the current situation.

\section{EXPERIMENTAL EVALUATIONS}

We now provide a detailed quantitative evaluation of VGRIS. First, we evaluated SLAaware scheduling in the case of underprovision of the GPU resource. Then, we studied the ability of proportional-share scheduling to maximize the GPU resource usage and the effectiveness of hybrid scheduling. We also evaluate VGRIS on heterogeneous virtualization platforms. Last, we performed some micro- and macroanalysis to evaluate VGRIS performance impact on the guest legacy software. 


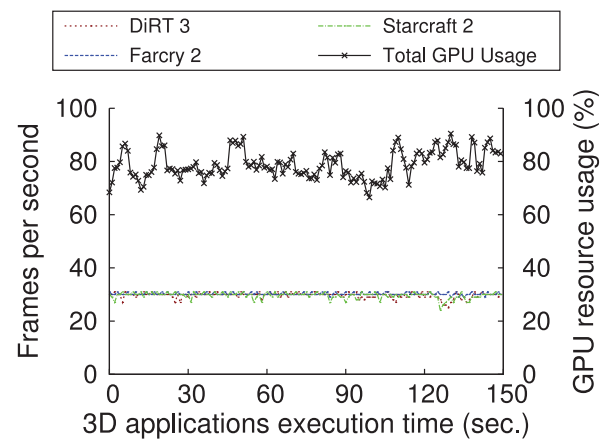

(a)

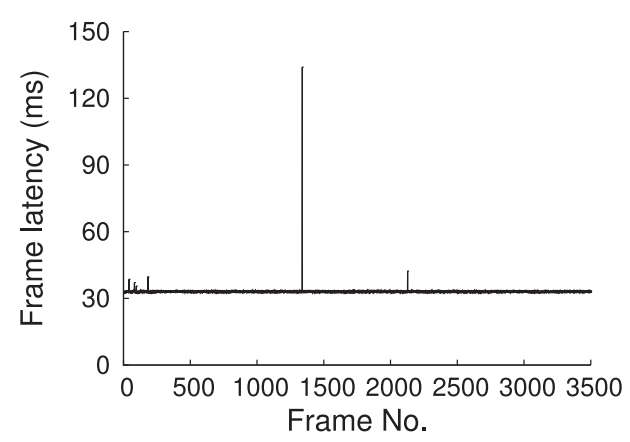

(b)

Fig. 10. SLA-aware scheduling results. (a) Frames Per Second (FPS) of DiRT 3, Farcry 2, and Starcraft 2. (b) Frame latency of Starcraft 2.

The testbed is configured with an i7-2600k 3.4GHz CPU, 16GB RAM, and an ATI HD6750 graphics card. Virtualization platforms consist of VirtualBox and VMware. Each hosted VM is configured with a Dual-Core CPU and 2GB RAM. Windows $7 \times$ 64 is used as both the host OS and guest OS. All games run at a high graphic quality (resolution 1,280 $\times 720$ ). To simplify the performance comparison, the swap space and th GPU-accelerated windowing system are disabled on the host side.

We used two different types of workload and one benchmark in the following experiments. The first workload group, named Ideal Model Games, has almost fixed objects and views, and hence a stable FPS is easily maintained. We choose PostProcess, LocalDeformablePRT, and so forth, from DirectX SDK samples to represent this kind of workload. The other group, named Reality Model Games, consists of games where the FPS rates vary frequently. We picked DiRT 3, Farcry 2, and Starcraft 2 as representative games.

We enhance the evaluations of our previous work [Yu et al. 2013] by evaluating VGRIS using three reality model games: DiRT 3, Farcry 2, and Starcraft 2. We also evaluate VGRIS on the heterogeneous virtualization platforms with VMs from VirtualBox and VMware. We are therefore able to prove that VGRIS is more practical and feasible in a cloud gaming scenario.

\subsection{SLA-Aware Scheduling Evaluation}

We first evaluate SLA-aware scheduling provided by VGRIS. We evaluate the algorithm using three workloads that are concurrently running on independent VMs, all from VMware, and sharing one single graphics card. The configurations are the same as the ones used for the experiments in Section 2. Figure 2 is the original performance when the three workloads, DiRT 3, Farcry 2, Starcraft 2, are concurrently running on the same GPU without the scheduling of VGRIS. Compared with it, Figure 10 shows the evaluation results of the SLA-aware scheduling using the same workloads. It highlights the scheduling effects. In Figure 10(a), the average FPS rates of DiRT 3, Starcraft 2, and Farcry 2 are 29.3, 30.4, and 30.1, respectively. All workloads are able to run under FPS rates that are smooth enough for users to play. The corresponding frame rate variances are $1.20,0.26$, and 1.36 . Hence, the SLA-aware scheduling algorithms can effectively control the FPS rates of the games. The percentage of excessive frame latency significantly drops to $0.20 \%$ in Figure $10(\mathrm{~b})$, with only one frame latency greater than $60 \mathrm{~ms}$, which is much better than the latency result of Figure 2(b). In addition, Figure 10(a) also shows the total GPU usage when employing SLA-aware scheduling. 


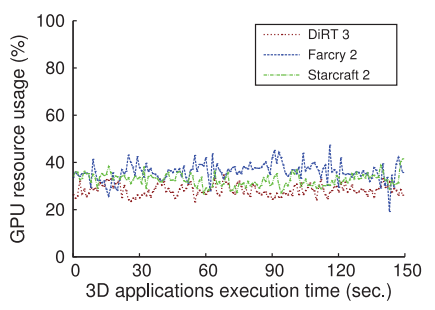

(a)

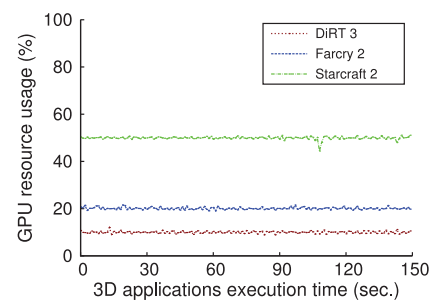

(b)

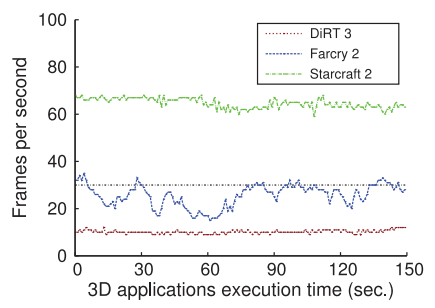

(c)

Fig. 11. Evaluation of GPU usage. (a) GPU usage results without proportional-share scheduling. (b) GPU usage with proportional-share scheduling. (c) Corresponding FPS results with proportional-share scheduling.

The maximum GPU usage is around 90\%, which means that the SLA-aware scheduling wastes GPU resources.

\subsection{Proportional-Share Scheduling Evaluation}

We next demonstrate the effectiveness of proportional-share scheduling in regulating the GPU resource usage according to the user settings. We also use the same reality model games and VMware as in the evaluation of SLA-aware scheduling. Figure 11(a) shows the original results of the GPU usage when VGRIS is not employed. From the figure, we can see that the GPU usage does not have regular patterns. Compared to this, Figure 11(b) shows the GPU resource usage of reality model games using different initial GPU shares: (1) DiRT 3 is set to use 10\% of the GPU resources, (2) Farcry 2 is set to use 20\%, and (3) Starcraft 2 uses 50\%. The results prove that proportional-share scheduling successfully provides a user-specified GPU resource share. Figure 11(c) shows the corresponding FPS results when proportional-share scheduling is leveraged in the system. Compared with the FPS rate results of the SLA-aware scheduling shown in Figure 10(a), the average FPS rates are 10.2 for DiRT 3, 25.6 for Farcry 2, and 64.7 for Starcraft 2. Despite that the total GPU usage is at a high level, two of the workloads still run at the FPS rate below 30. The corresponding frame rate variances are 0.57 , 21.99 , and 4.39, respectively. From the data, we can see that the proportional-share cannot always guarantee the SLA requirements of all games. The fluctuations of the FPS rates of the three games are also larger when SLA-aware scheduling is used.

\subsection{Hybrid Scheduling Evaluation}

We now evaluate hybrid scheduling automatic scheduling determination algorithms with the proper parameters. The same workloads and VMware VMs in the evaluations of the former two algorithms were used to evaluate hybrid scheduling effectiveness: DiRT 3, Farcry 2, and Starcraft 2. Figure 12 illustrates the algorithm selections and their impacts on the FPS of the running VMs. In this experiment, we set FPSthres to 30, GPUthres to $85 \%$, and Time to 5 seconds. We first run three games concurrently. Hybrid scheduling uses SLA-aware scheduling because of the low frame rate in the loading screen. After 5 seconds, hybrid scheduling detects a low overall GPU usage over the past 5 seconds. As a result, it employs the proportional-share scheduling algorithm and equally allocates redundant GPU resources to each VM, which results in a performance increase in the rendering of the upcoming frame. After 10 seconds, the VM running DiRT 3 did not benefit from sufficient GPU resources to maintain its SLA over the past 5 seconds. Hence, hybrid scheduling employs SLA-aware scheduling to release excessive GPU resources from other VMs. Then, after 5 more seconds, hybrid scheduling switches back to proportional-share scheduling. It was found that DiRT 3 FPS decreases when the proportional-share scheduling algorithm was selected. This is caused by the low redundant GPU resource. Moreover, note that real-world games tend 


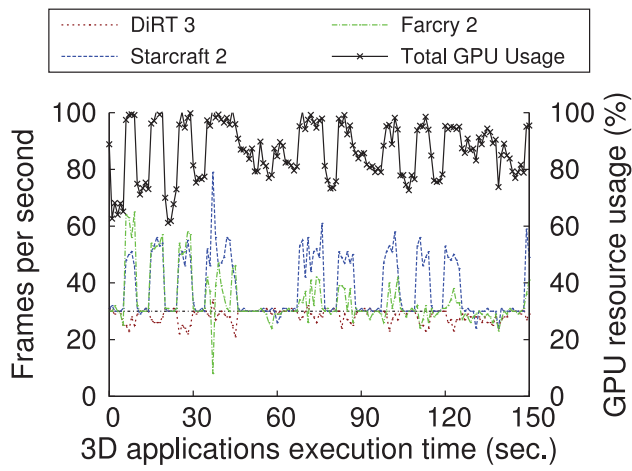

Fig. 12. Evaluation results of hybrid scheduling algorithm.

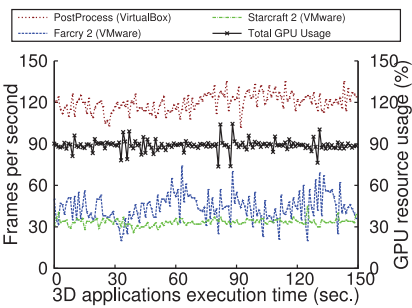

(a)

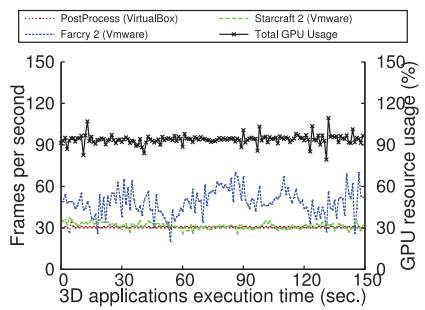

(b)

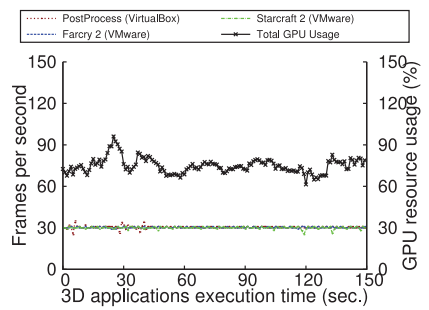

(c)

Fig. 13. Evaluation of VGRIS on hybrid platforms with PostProcess running in VirtualBox, and Farcry 2 and Starcraft 2 running in VMware. (a) Original results without scheduling. (b) Evaluation results with SLA-aware scheduling applied to VirtualBox and no scheduling applied to VMware. (c) Evaluation results with SLA-aware scheduling applied to both VirtualBox and VMware.

to have a varied complexity of game scenes quickly varying, and as such the amount of GPU resources required during the whole runtime can change greatly.

In Figure 12, the average FPS rates are 29.0 for DiRT 3, 38.2 for Farcry 2, and 33.4 for Starcraft 2 when the hybrid scheduling is applied. The SLA requirements of all three games are basically satisfied. The frame rate variances are 5.38, 115.14, and 76.05. In our analysis, the frequent switches between the SLA-aware scheduling and proportional-share scheduling result in the abrupt changes, as well as the large fluctuations of the FPS rates.

\subsection{Evaluation on Heterogeneous Platforms}

To prove that VGRIS can be employed under various virtualization platforms, we conducted the experiments on the heterogeneous platforms with one VM from VirtualBox and two VMs from VMware, shown in Figure 13. Since VirtualBox is not compatible with the games implemented using some advanced and complex features from the 3D graphics libraries, as discussed in Section 4.1, we select PostProcess, a benchmark from DirectX SDK to run inside the VM of VirtualBox. Farcry 2 and Starcraft 2 that run inside the VMs of VMware are configured the same as in the previous experiments.

Figure 13(a) shows the original results of FPS and GPU usage when VGRIS is not leveraged to schedule the GPU resources. PostProcess in VirtualBox runs at an average FPS rate of 119. When the SLA-aware scheduling is applied only to VirtualBox, the FPS rate of PostProcess remains at 30, as shown in Figure 13(b). Without any scheduling from VGRIS, in Figure 13(b), Farcry 2 and Starcraft 2 run at their original FPS rate, which are virtually the same as in Figure 13(a). In Figure 13(c), the 


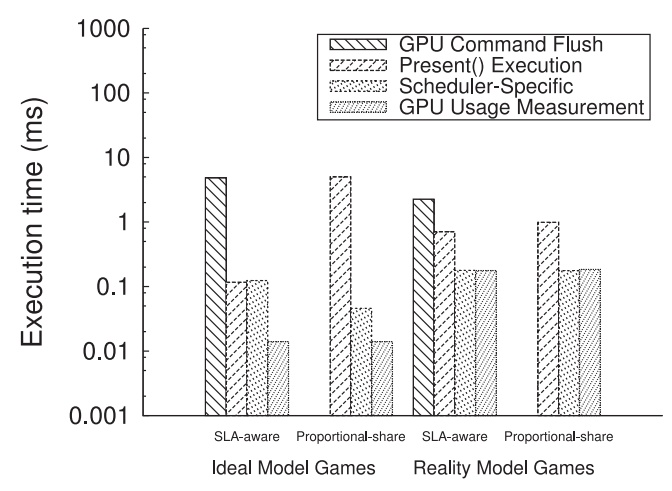

Fig. 14. Microbenchmark results.

Table III. Macrobenchmark Evaluation

\begin{tabular}{|l||c|c|c|c|c|}
\hline \multicolumn{1}{|c||}{} & Native & \multicolumn{2}{c|}{ SLA-Aware Scheduling } & \multicolumn{2}{c|}{ Proportional-Share Scheduling } \\
\cline { 2 - 6 } & FPS & FPS & Overhead & FPS & Overhead \\
\hline \hline DiRT 3 & 68.61 & 66.86 & $2.55 \%$ & 67.35 & $1.84 \%$ \\
Starcraft 2 & 67.58 & 64.01 & $5.28 \%$ & 64.59 & $4.42 \%$ \\
Farcry 2 & 90.42 & 89.48 & $1.04 \%$ & 86.34 & $4.51 \%$ \\
\hline
\end{tabular}

SLA-aware scheduling is applied to all VMs, resulting in all workloads running at the FPS rate of 30 .

In our experiments, the same evaluation results as in Figures 11 and 12 are achieved when the proportional-share scheduling and hybrid scheduling are applied to the heterogeneous platforms of VirtualBox and VMware. As a consequence, VGRIS is also feasible and practical on VirtualBox in addition to VMware.

\subsection{Performance Discussions}

To evaluate the performance impact of VGRIS on legacy applications and OS, we first performed microanalysis to illustrate the potential hot spot. PostProcess and DiRT 3 are leveraged to utilize available GPU resources in this experiment. We only evaluate the execution cost of each part in SLA-aware scheduling and proportionalshare scheduling. Hhybrid scheduling is not included because there are only trivial changes based on the other two scheduling methods, and the performance impact can be ignored. Figure 14 shows the microbenchmark results. The execution time of SLAaware scheduling constitutes four parts, in which the GPU command flush operations contribute the main performance overhead. This is due to the design of the current Direct3D library and the implemented flush strategy in the VGRIS prototype. It is possible to achieve a better result by adopting different flush strategies in the future.

Having no GPU Command Flush operation, proportional-share scheduling contains three parts in its execution time. For the same reason, the Present API execution time unsurprisingly becomes the most expensive operation. It is noteworthy that no aggressive flush of the Direct3D command buffer is added in proportional-share scheduling, because proportional-share scheduling always assumes the existence of overprovision in the GPU resource. In total, the SLA-aware scheduling algorithm incurs $2.47 \%$ of the execution time overhead of the native function for PostProcess and $162.58 \%$ for DiRT 3. The results for proportional-share scheduling are $1.77 \%$ and $6.56 \%$.

Considering the infrequent invocation of Present API, the performance overhead is significantly decreased. Table III shows the evaluation results, proving that VGRIS brings in $2.96 \%$ performance overhead on average for SLA-aware scheduling and 3.59\% 
on average for proportional-share scheduling. Thus, the scheduling methods provided by VGRIS are demonstrated to incur slight performance overhead. As a result, VGRIS is able to execute multiple game VMs concurrently while ensuring acceptable SLAs individually.

\section{RELATED WORK}

Virtualized resource management has been an active research area over the past decade. Based on the general trend of all related research works, we can broadly classify them into three groups: (1) scheduling in virtualization, (2) GPU scheduling, and (3) applications of GPU virtualization.

Scheduling in virtualization. Previous works focused on CPU and I/O schedulings. Credit, Simple Earliest Deadline First (SEDF), and Borrowed Virtual Time (BVT) [Duda and Cheriton 1999] are some CPU schedulers [Cherkasova et al. 2007] for general-purpose hypervisors like Xen [Barham et al. 2003]. Scheduling the CPU resources according to the indicated proportions, these methods can also be employed in the proportional-share scheduling in VGRIS. BVT is optimized for latency-sensitive applications, as it reduces the corresponding scheduling time of the next job and borrows time slices from its future CPU usage. Credit scheduling achieves the same optimization by boosting the corresponding virtual CPU in the block state when an external event arrives [Cherkasova et al. 2007]. Besides, the CPU schedulers for real-time guest OS control the expected latency by arranging the virtual CPU run queue in a certain order [Lee et al. 2010; Yu et al. 2010]. However, all of these scheduling methods cannot be applied to manage GPU resources to fulfill the SLA requirement. The reason is that all of these scheduling approaches treat the VM as a black box and hence ignore the SLA-related measurements of the guest applications. In contrast, by effectively intercepting library APIs on the host side, VGRIS can perform SLA-aware scheduling without modifying the guest software.

GPU scheduling. Previous GPU resource scheduling approaches mainly targeted native systems. For example, Wang et al. [2011] employed three strategies to apply power gating on GPUs. Elliott and Anderson [2012] presented two methods for integrating GPUs into soft real-time multiprocessor systems to improve the overall system performance. They also proposed GPUSync [Elliott et al. 2013], in which there are both staticand dynamic-priority GPU schedulers that deal with multi-GPU multicore real-time systems. However, VGRIS is not a real-time system. It tries to focus on the balance of the GPU usage while fully utilizing the GPU resources. A task-based dynamic loadbalancing scheduling [Chen et al. 2010] is proposed for single and multi-GPU systems. Ravi et al. [2011] proposed a framework that enables applications running within VMs to transparently share one or more GPUs. In comparison, VGRIS is only able to share resources of one graphics card for VMs. It will be extended to enable VMs to share more GPUs by the technologies of these prior works. Malits et al. [2012] analyzed the root of the inefficiencies of the control flow of CUDA applications and tried to eliminate them. Compared to their work, VGRIS mainly focuses on graphics processing including 3D rendering and gaming. Both the SLA of the 3D applications in the VMs and the overall throughput are taken into account.

Kato et al. [2011a] addressed the priority inversion problems of the user GPU tasks in GPU-accelerated windowing systems. Similar to VGRIS, applying their solutions requires no modifications to the $\mathrm{X}$ server or user applications. TimeGraph [Kato et al. 2011b], also proposed by Kato et al., implements a real-time GPU scheduler to isolate performance for important GPU workloads. To achieve its design goal, TimeGraph modifies the codes at the device driver level. TimeGraph cannot guarantee compatibility 
for all platforms. Instead, VGRIS is a lightweight scheduler. Employing our framework requires no modification to OS, device drivers, and applications. Kato et al. [2012] also proposed Gdev. Gdev is an ecosystem of GPU resource management that uses GPUs as first-class computing resources. There is a scheduling scheme, focusing on isolation among working sets of multitasking system. However, VGRIS leverages the virtualization technology so that it in itself can isolate the GPU resources for different VMs.

Rossbach et al. [2011] proposed PTask, which is a framework for first-class computing resources. This architecture inserts an API layer connecting user-mode programs to OS kernel, providing a dataflow programming model. Their work is similar to VGRIS, in which an interposition API is proposed to intercept graphics libraries from VMs to the host OS. Becchi et al. [2012] added two features to improve GPU sharing: dynamic application-to-GPU binding and virtual memory for GPUs. Aimed at different goals, VGRIS can further employ this work to support load balancing and solve GPU memory constraints for applications.

GERM [Bautin et al. 2008] aimed at providing fair GPU resource allocation. Besides, fixed frame rate approaches like Vertical Synchronization (V-Sync) ${ }^{3}$ are designed for games to avoid an excessive use of the hardware resource. Unfortunately, GERM fails to consider the SLA requirements, and fixed frame rate approaches fail to consider the effective use of the hardware resources. Due to a fixed frame rate, both approaches prevent an on-the-fly adjustment of the resources. VGRIS can guarantee SLA requirements while making full use of the excessive GPU resources.

Applications of GPU virtualization. Currently, NVIDIA provides GRID ${ }^{4}$ to virtualize GPU on hardware. This improvement would enable rich graphics in virtualized environments, which means that multiple users can share a single GPU, improving user density while providing true PC performance and compatibility. The rapid development of GPU virtualization generates many new uses, especially in cloud gaming and GPGPU computing.

Previous studies on the cloud gaming platform focus on streaming graphical content and decreasing the required network bandwidth [Noimark and Cohen-Or 2003; Jurgelionis et al. 2009]. Li et al. [2011] use cryo-electron microscopy 3D reconstruction as an example to present how to exploit parallelism on both CPUs and GPUs in a heterogeneous system. However, our approach, based on the GPU paravirtualization technique, is able to run multiple games on VMs sharing the GPU resources.

Lutz et al. [2013] focused on GPGPU for stencil applications. They optimized the distribution of the application to increase its performance. vCUDA [Shi et al. 2009] introduced GPU computing into a virtualized execution environment. It motivates our research in scheduling resources for GPU computing. rCUDA Duato et al. [2010] and other work of Duato et al. [2009] try to decrease the power consumption of the GPUs from high performance clusters while preserving their 3D acceleration capability on the remote nodes. Gupta et al. [2011] proposed Pegasus using some NVIDIA GPGPUs coupled with some $\mathrm{x} 86$-based general-purpose host cores to manage combined platform resources. Based on Pegasus, Merritt et al. [2011] proposed Shadowfax, a prototype of GPGPU assemblies, improved GPGPU application scalability as well as increased the application throughput. Yang et al. [2012] proposed a compiler for GPGPU to optimize applications in two ways: one is to effectively utilize the GPU memory and the other is to manage parallelism.

${ }^{3}$ V-Sync: http://en.wikipedia.org/wiki/Vertical_synchronization/.
${ }^{4}$ NVIDIA GRID: http://www.nvidia.com/object/grid-boards.html. 
However, none of these approaches has studied the management of virtualized GPU resource isolation and scheduling to achieve computational efficiency, which is the main focus of VGRIS. Compared to other approaches, our approach improves the SLA of the GPU computation on cloud platform and maximizes the overall resource usage. Additionally, VGRIS provides three representative scheduling algorithms to meet multiple optimization goals in case of under- and overprovisioned GPU resources.

\section{CONCLUSION}

We presented VGRIS, a virtualized GPU resource isolation and scheduling framework for GPU-related computational tasks. By introducing an agent per VM and a centralized scheduling controller to the paravirtualization framework, VGRIS achieves in-VM GPU resource measurements and regulates the GPU resource usage. Moreover, we extended VGRIS with a set of APIs to make VGRIS able to host a variety of scheduling algorithms without modifying the framework itself. Three representative scheduling algorithms are implemented by the API: SLA-aware scheduling, which allocates just enough GPU resources to fulfill the SLA requirement; proportional-share scheduling, which allocates all GPU resources to all running VMs in proportion to their weights; and hybrid scheduling, which provides a mixed solution to meet the SLA requirement while maximizing the overall GPU resource usage. Using the cloud gaming scenario as a case study, our evaluation demonstrates that each scheduling algorithm fulfills its goals under various workloads. The experimental results on heterogeneous virtualization platforms show that VGRIS is adapted to different VMs. As a future work, we plan to extend VGRIS to multiple physical GPUs and multiple physical machine systems for data center resource scheduling.

\section{ACKNOWLEDGMENTS}

Thanks to Jiewei Wu and Xi Chen for their contributions to this project. We also thank Yueqiang Cheng and Zheng Zhang for their suggestions. In addition, we appreciate the valuable comments from our reviewers. They helped us revise our work one step further.

\section{REFERENCES}

Paul Barham, Boris Dragovic, Keir Fraser, Steven Hand, Timothy L. Harris, Alex Ho, Rolf Neugebauer, Ian Pratt, and Andrew Warfield. 2003. Xen and the art of virtualization. In Proceedings of the ACM Symposium on Operating Systems Principles (SOSP).

Mikhail Bautin, Ashok Dwarakinath, and Tzi cker Chiueh. 2008. Graphic engine resource management. In Proceedings of Multimedia Computing and Networking (MMCN).

Michela Becchi, Kittisak Sajjapongse, Ian Graves, Adam Procter, Vignesh Ravi, and Srimat Chakradhar. 2012. A virtual memory based runtime to support multi-tenancy in clusters with GPUs. In Proceedings of the International Symposium on High-Performance Parallel and Distributed Computing (HPDC).

Long Chen, Oreste Villa, Sriram Krishnamoorthy, and Guang R. Gao. 2010. Dynamic load balancing on single- and multi-GPU systems. In Proceedings of the IEEE International Symposium on Parallel Distributed Processing (IPDPS).

Ludmila Cherkasova, Diwaker Gupta, and Amin Vahdat. 2007. Comparison of the three CPU schedulers in Xen. SIGMETRICS Performance Evaluation Review 35, 2, 42-51.

Micah Dowty and Jeremy Sugerman. 2009. GPU virtualization on VMware's hosted I/O architecture. SIGOPS Operating Systems Review 43, 3, 73-82.

José Duato, Francisco D. Igual, Rafael Mayo, Antonio J. Peña, Enrique S. Quintana-Ortí, and Federico Silla. 2009. An efficient implementation of GPU virtualization in high performance clusters. In Proceedings of the European Conference on Parallel Processing (Euro-Par Workshops).

José Duato, Antonio J. Peña, Federico Silla, Rafael Mayo, and Enrique S. Quintana-Ortí. 2010. rCUDA: Reducing the number of GPU-based accelerators in high performance clusters. In Proceedings of the International Conference on High Performance Computing and Simulation (HPCS). 
Kenneth J. Duda and David R. Cheriton. 1999. Borrowed-virtual-time (BVT) scheduling: Supporting latencysensitive threads in a general-purpose scheduler. In Proceedings of the ACM Symposium on Operating Systems Principles (SOSP).

Glenn A. Elliott and James H. Anderson. 2012. Globally scheduled real-time multiprocessor systems with GPUs. Real-Time Systems 48, 1, 34-74.

Glenn A. Elliott, Bryan C. Ward, and James H. Anderson. 2013. GPUSync: A framework for real-time GPU management. In Proceedings of the Real-Time Systems Symposium (RTSS).

Vishakha Gupta, Ada Gavrilovska, Karsten Schwan, Harshvardhan Kharche, Niraj Tolia, Vanish Talwar, and Parthasarathy Ranganathan. 2009. GViM: GPU-accelerated virtual machines. In Proceedings of the ACM Workshop on System-Level Virtualization for High Performance Computing (HPCVirt).

Vishakha Gupta, Karsten Schwan, Niraj Tolia, Vanish Talwar, and Parthasarathy Ranganathan. 2011. Pegasus: Coordinated scheduling for virtualized accelerator-based systems. In Proceedings of the USENIX Annual Technical Conference (ATC).

Joystiq. 2009. GDC09 Interview: OnLive Founder Steve Perlman, Continued. Retrieved June 13, 2014, from http://www.joystiq.com/2009/04/02/gdc09-interview-onlive-founder-steve-perlman-continued/.

Audrius Jurgelionis, Philipp Fechteler, Peter Eisert, Francesco Bellotti, Haggai David, Jukka-Pekka Laulajainen, Richard Carmichael, Vassilis Poulopoulos, Arto Laikari, Pekka H. J. Perälä, Alessandro De Gloria, and Christos Bouras. 2009. Platform for distributed 3D gaming. International Journal of Computer Games Technology 2009, Article 1.

Shinpei Kato, Karthik Lakshmanan, Yutaka Ishikawa, and Ragunathan (Raj) Rajkumar. 2011a. Resource sharing in GPU-accelerated windowing systems. In Proceedings of the 17th IEEE Real-Time and Embedded Technology and Applications Symposium (RTAS).

Shinpei Kato, Karthik Lakshmanan, Ragunathan Rajkumar, and Yutaka Ishikawa. 2011b. TimeGraph: GPU scheduling for real-time multi-tasking environments. In Proceedings of the USENIX Annual Technical Conference (ATC).

Shinpei Kato, Michael McThrow, Carlos Maltzahn, and Scott Brandt. 2012. Gdev: First-class GPU resource management in the operating system. In Proceedings of the USENIX Annual Technical Conference (ATC).

H. Andrés Lagar-Cavilla, Niraj Tolia, Mahadev Satyanarayanan, and Eyal de Lara. 2007. VMM-independent graphics acceleration. In Proceedings of the International Conference on Virtual Execution Environments (VEE).

Min Lee, A. S. Krishnakumar, P. Krishnan, Navjot Singh, and Shalini Yajnik. 2010. Supporting soft real-time tasks in the Xen hypervisor. In Proceedings of the 6th ACM SIGPLAN / SIGOPS International Conference on Virtual Execution Environments (VEE).

Linchuan Li, Xingjian Li, Guangming Tan, Mingyu Chen, and Peiheng Zhang. 2011. Experience of parallelizing cryo-EM 3D reconstruction on a CPU-GPU heterogeneous system. In Proceedings of the ACM International Symposium on High Performance Distributed Computing (HPDC).

Thibaut Lutz, Christian Fensch, and Murray Cole. 2013. PARTANS: An autotuning framework for stencil computation on multi-GPU systems. ACM Transactions on Architecture and Code Optimization 9, 4, Article 59.

Roman Malits, Evgeny Bolotin, Avinoam Kolodny, and Avi Mendelson. 2012. Exploring the limits of GPGPU scheduling in control flow bound applications. ACM Transactions on Architecture and Code Optimization 8, 4, Article 29.

Alexander M. Merritt, Vishakha Gupta, Abhishek Verma, Ada Gavrilovska, and Karsten Schwan. 2011. Shadowfax: Scaling in heterogeneous cluster systems via GPGPU assemblies. In Proceedings of the 5th International Workshop on Virtualization Technologies in Distributed Computing (VTDC).

Beng Heng Ng, Billly Lau, and Atul Parkash. 2009. Direct Access to Graphics Card Leveraging VT-d. Technical Report. University of Michigan.

Yuval Noimark and Daniel Cohen-Or. 2003. Streaming scenes to MPEG-4 video-enabled devices. IEEE Computer Graphics and Applications 23, 1, 58-64.

John D. Owens, Mike Houston, David Luebke, Simon Green, John E. Stone, and James C. Phillips. 2008. GPU computing. Proceedings of the IEEE 96, 5, 879-899.

Rajat Phull, Cheng-Hong Li, Kunal Rao, Srihari Cadambi, and Srimat T. Chakradhar. 2012. Interferencedriven resource management for GPU-based heterogeneous clusters. In Proceedings of the ACM International Symposium on High Performance Distributed Computing (HPDC).

Vignesh T. Ravi, Michela Becchi, Gagan Agrawal, and Srimat T. Chakradhar. 2011. Supporting GPU sharing in cloud environments with a transparent runtime consolidation framework. In Proceedings of the ACM International Symposium on High Performance Distributed Computing (HPDC). 
Christopher J. Rossbach, Jon Currey, Mark Silberstein, Baishakhi Ray, and Emmett Witchel. 2011. PTask: Operating system abstractions to manage GPUs as compute devices. In Proceedings of the ACM Symposium on Operating Systems Principles (SOSP).

Lin Shi, Hao Chen, and Jianhua Sun. 2009. vCUDA: GPU accelerated high performance computing in virtual machines. In Proceedings of the IEEE International Symposium on Parallel Distributed Processing (IPDPS).

Po-Han Wang, Chia-Lin Yang, Yen-Ming Chen, and Yu-Jung Cheng. 2011. Power gating strategies on GPUs. ACM Transactions on Architecture and Code Optimization 8, 3, Article 13.

Yin Wang and Arif Merchant. 2007. Proportional-share scheduling for distributed storage systems. In Proceedings of the 5th Conference on File and StorageTechnologies (FAST).

Yi Yang, Ping Xiang, Jingfei Kong, Mike Mantor, and Huiyang Zhou. 2012. A unified optimizing compiler framework for different GPGPU architectures. ACM Transactions on Architecture and Code Optimization 9, 2, Article 9.

Miao Yu, Chao Zhang, Zhengwei Qi, Jianguo Yao, Yin Wang, and Haibing Guan. 2013. VGRIS: Virtualized GPU resource isolation and scheduling in cloud gaming. In Proceedings of the International Symposium on High-Performance Parallel and Distributed Computing (HPDC).

Peijie Yu, Mingyuan Xia, Qian Lin, Min Zhu, Shang Gao, Zhengwei Qi, Kai Chen, and Haibing Guan. 2010. Real-time enhancement for Xen hypervisor. In Proceedings of Embedded and Ubiquitous Computing (EUC).

Received August 2013; revised January 2014; accepted January 2014 